\title{
COMPARITIVE ANALYSIS OF DOA AND BEAMFORMING ALGORITHMS FOR SMART ANTENNA SYSTEMS
}

\author{
Amrita Soni ${ }^{1}$, Mukesh Wadhwani ${ }^{2}$ \\ ${ }^{I}$ Asst. Professor, Electronics and Communication Engg dept, UEC, Ujjain, M.P, India \\ ${ }^{2}$ Student, Electronics and Communication Engg dept, UEC, Ujjain, M.P, India
}

\begin{abstract}
This paper revolves around the implementation of Direction of arrival and Adaptive beam-forming algorithms for Smart Antenna Systems. This paper also investigates the implementation of algorithms on various planner array geometries viz. circular and rectangular. Music algorithm is primarily finds the possible location of desired user and adaptive beam-forming algorithms such as LMS, RLS and CMA algorithms adapts the weights of the array. DOA estimation gives the maximum peak of spectrum with respect to angle of arrival where the desired user is supposed to exist. After DOA estimation weights of array antenna are changed with the changing received signal. This methodology is called as Spectral estimation, which allows the antenna pattern to steer in desired direction estimated by DOA and simultaneously null out the interfering signals. Rate of convergence is the major criterion for comparison for adaptive beam-forming algorithms.
\end{abstract}

Keywords: DOA, MUSIC, LMS, RLS, CMA, SAS. $* * *$

\section{INTRODUCTION}

Due to increasing demands for higher data rate and growing user base in a limited allotted spectrum, there exist need for Smart signal processing which is possible through Smart Antenna [1].The antenna array usually do not adapt to the time changing Electromagnetic environment. The futuristic smartness makes the antenna array to form the radiation pattern in the desired direction. This is called as the adaptive beam-forming $(\mathrm{ABF})$. This is done via digital signal processing, thus also called digital beam-forming (DBF).

Figure 1.1 shown below is a two stage block diagram which shows Smart antenna systems SAS with features of Direction of Arrival DOA estimation in the first stage and in the second stage Adaptive beam-forming network with dynamic weight updates. This figure also suggest the application of both stage algorithms with linear or planner array geometries.

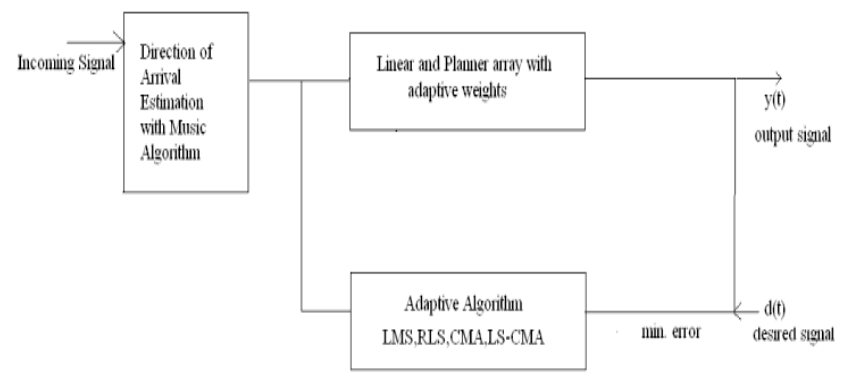

Fig- 1.1: Block Diagram of SAS with DOA and Adaptive Beam-forming Together.

Research shows the implementation of two dimensional antenna array for handling massive MIMO configurations which will improve the performance in large user base situation [3]. Figure 1.2 also shows a conceptual model where instead of linear array on the base station planner array is shown.

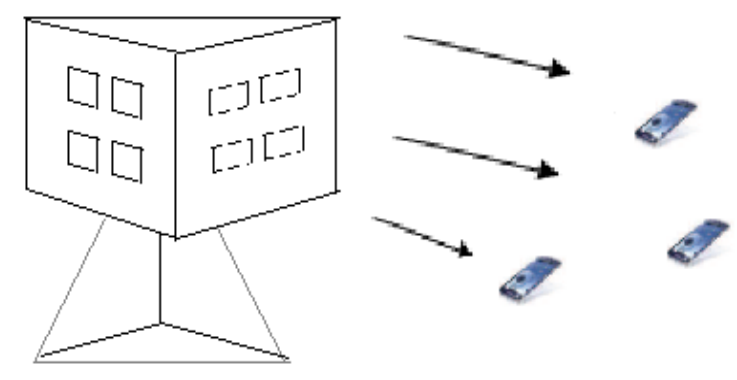

Fig-1.2: Conceptual 2D Antenna array geometry for implementation of SAS leading to handle multiple user base.

A single source or transmitter whether constant or moving may have many propagation paths and angle of arrival, due to the effects like reflection, refraction, scattering and dispersion of Electromagnetic signal. It becomes much important when there is large number of transmitters to estimate angle of arrival. There exist various Angle of arrival methods. Godara[12], suggested three different categories, Spectrum based, Parametric based, and subspace fitting based. Spectrum based are easier in implementation namely these are Capon AOA, and MUSIC multiple signal classification (spectrum with subspace) which is a high resolution method which gives the graph for pseudo spectrum and angle of arrival [12]. Practical condition of application involves the effect of mutual coupling which can be resolved with proper steering vector [4]. This study works out with Music algorithm for uniform linear array and uniform circular array. However it can also be employed for rectangular planner array. 
Another stage of Smart antenna system is the Adaptive beam forming algorithms. Smartness lies in shaping the beam according to certain conditions of channel and receiver. This also controls the performance of antenna. There exists some parameter for beam-forming such as maximizing SIR signal to interference ratio, minimizing noise variance, minimizing mean square error, steering towards signal of interest, nulling or reducing interference and tracking moving transmitters [14]. Phase shifting and dynamic array weighting are premiere objectives of DBF algorithm. Some of DBF algorithms are Least Mean Square LMS, Recursive Least Square RLS, Constant Modulus Algorithm CMA and LS CMA, CGA are named a few. All are iterative algorithms so number of iterations and convergence rate are the comparison criterion. This paper deals with LMS, RLS and CMA with their comparison and simulation result.

\section{DOA AND ADAPTIVE BEAM FORMING}

As Discussed above DOA and Adaptive Beam forming are integral parts of the SAS.

\subsection{Direction of Arrival}

Due to multipath propagation effects, received signal is impinge on the receiving antenna from different directions.DOA is an important property for characterizing channel [12]. Estimation of direction of arrival can be helpful at both uplink and downlink processing in mobile communication. Spectrum based methods are mainly discussed such as Music algorithm.

DOA algorithm (Music) can be understood with help of given figure.

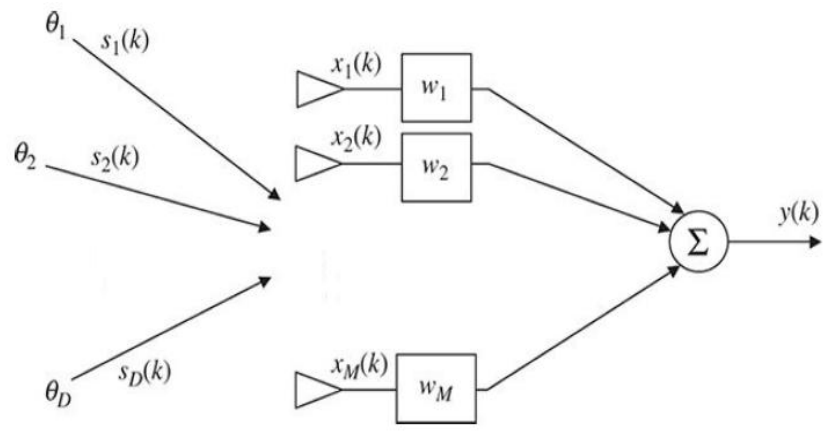

Fig- 2: Direction of arrival estimation with D signal towards $\mathrm{M}$ array of antenna with $\mathrm{w}$ as the weight of the antenna.

As the above figure shows $\mathrm{D}$ signals arrive from $\mathrm{D}$ directions at $M$ elements array with $\mathrm{x}(\mathrm{k})$ is the received signal, $\mathrm{s}(\mathrm{k})$ is received signal, $\mathrm{a}(\Theta)$ is the steering vector with $\mathrm{n}(\mathrm{k})$ as AWGN additive white Gaussian noise and $\mathrm{k}$ are the discrete time sampling instants.

Output of array is $\mathrm{y}(\mathrm{k})=\mathrm{w}^{\mathrm{T}} * \mathrm{x}(\mathrm{k})$

$\mathrm{x}(\mathrm{k})=\mathrm{a}_{\mathrm{i}}(\Theta) * \mathrm{~s}_{\mathrm{i}}(\mathrm{k})+\mathrm{n}(\mathrm{k})$ where $\mathrm{i}$. is from 1 to $\mathrm{D}$
DOA method are worked out with correlation matrices and correlation matrix of input signal $\mathrm{x}$ is calculated as,

$$
\mathrm{R}_{\mathrm{xx}}=\mathrm{E}\left[\mathrm{x} * \mathrm{x}^{\mathrm{H}}\right]=\mathrm{a} * \mathrm{R}_{\mathrm{ss}} * \mathrm{a}^{\mathrm{H}}+\mathrm{R}_{\mathrm{nn}}
$$

where $R_{s s}$ and $R_{n n}$ are received signal and noise correlation. As the noise is AWGN, $\mathrm{R}_{\mathrm{nn}}$ is given as $\sigma^{2} * \mathrm{I}$ with $\mathrm{I}$ as $\mathrm{M} * \mathrm{M}$ identity matrix.

This subspace DOA method in above equation of input signal $\mathrm{x}(\mathrm{k})$ in eigen vector matrix Es as $M^{*} \mathrm{D}$ signal subspace and eigen vector matrix En as $M^{*}(M-D)$ in noise subspace. For every DOA Pseudo spectrum $\mathrm{P}(\Theta)$ is calculated which gives maximum spectrum peak with desired angle of arrival. Pseudo spectrum based on desired angle of arrival $\Theta$ is given as

$$
\mathrm{P}_{\mathrm{mu}}(\Theta)=1 / \mid\left(\mathrm{a}(\Theta) * \mathrm{En}^{*} \mathrm{En}^{\mathrm{H}} * \mathrm{a}(\Theta)^{\mathrm{H}} \mid\right.
$$

Music method is a high resolution threshold method. Its resolution depends on SNR and number of elements [12].

Where as Conventional DOA approach do not uses subspace approach that is why it has lower resolution.

$$
\mathrm{P}_{\text {con }}(\Theta)=\left|\mathrm{a}^{\mathrm{H}}(\Theta) * \mathrm{Rrr} * \mathrm{a}(\Theta)\right| / \mid\left(\mathrm{a}(\Theta)^{\mathrm{H}} * \mathrm{a}(\Theta) \mid[12]\right.
$$

\subsection{Adaptive Beam-Forming}

There exist fixed beam forming methods such as maximum likelihood and minimum variance [14] but are not useful when the angle of arrival changes with time. As the angle of arrival keeps on changing with time due to multipath effects array weights should be optimized. LMS, RLS and CMA are analyzed and simulated in this paper.

In Least Mean square method, minimum mean square error MSE is calculated [7]. This error is actually the difference between desired signal $\mathrm{d}(\mathrm{k})$ which is a prior estimated signal and of weight $\mathrm{w}(\mathrm{k})$ and input signal $\mathrm{x}(\mathrm{k})$ product as,

$$
\begin{aligned}
& \xi(\mathrm{k})=\text { error signal }=\mathrm{d}(\mathrm{k})-\mathrm{w}^{\mathrm{H}} * \mathrm{x}(\mathrm{k}) \\
& |\xi(\mathrm{k})|^{2}=\left|\mathrm{d}(\mathrm{k})-\mathrm{w}^{\mathrm{H}} * \mathrm{x}(\mathrm{k})\right|^{2}
\end{aligned}
$$

Equation 6 shows mean square error it should be minimum with respect to iterations.

Moreover signal characteristics are not known thus input signal correlation matrix is involved in the calculation.

$$
\begin{aligned}
& \mathrm{R}_{\mathrm{xx}}(\mathrm{k})=\mathrm{x}(\mathrm{k}) * \mathrm{x}^{\mathrm{H}}(\mathrm{k})=\text { array correlation matrix } \\
& \mathrm{r}(\mathrm{k})=(\mathrm{d} *(\mathrm{k})) * \mathrm{x}(\mathrm{k})=\text { signal correlation vector }
\end{aligned}
$$

Now, iterative approximation for array weights is required

$$
\mathrm{w}(\mathrm{k}+1)=\mathrm{w}(\mathrm{k})-0.5 * \mu * \mathbb{v}\left(\mathrm{R}_{\mathrm{xx}} * \mathrm{w}-\mathrm{r}\right)
$$

$\mathbb{v}$ is gradient function 
The convergence of LMS algorithm is dependent on stepsize parameter $\mu$ which in our simulation is between 0 and 1 .

For proper convergence it is given by $0 \leq \mu \leq 1 / \lambda_{\max }$.

In Recursive least square method computational efficiency is improved by using $\mathrm{k}$ time sample block and calculating correlation matrix over that time block. This is called as the recursive calculation. RLS is improvised by using a forgetting factor alpha unlike step-size parameter of LMS algorithm. This factor alpha between 0 and 1 deemphasize the earlier samples and emphasize the most recent ones[14].

So by RLS algorithm recursive calculation of correlation matrix is done which improves the computational efficiency.

$$
\begin{aligned}
& \text { Mathematically, } \operatorname{Rxx}(\mathrm{k})=\Sigma_{\mathrm{i}=1}^{\mathrm{k}}\left[\alpha^{\mathrm{k}-\mathrm{i}} * \mathrm{x}(\mathrm{i}) * \mathrm{x}^{\mathrm{H}}(\mathrm{i})\right] \\
& \mathrm{r}(\mathrm{k})=\Sigma_{\mathrm{i}=1}^{\mathrm{k}}\left[\alpha^{\mathrm{k}-\mathrm{i}} *\left(\mathrm{~d}^{*}(\mathrm{i})\right)^{*} \mathrm{x}(\mathrm{i})\right]
\end{aligned}
$$

Weight update is given by

$$
\begin{aligned}
& \mathrm{W}(\mathrm{k})=\mathrm{w}(\mathrm{k}-1)+\mathrm{g}(\mathrm{k}) *\left[\mathrm{~d} *(\mathrm{k})-\mathrm{x}^{\mathrm{H}}(\mathrm{k}) * \mathrm{w}(\mathrm{k}-1)\right] \\
& \mathrm{g}(\mathrm{k})=\mathrm{R}_{\mathrm{xx}}(\mathrm{k})^{-1} * \mathrm{r}(\mathrm{k})
\end{aligned}
$$

It has much faster convergence as compared to LMS algorithm. One thing to be noticed is $\mathrm{d}(\mathrm{t})$ and $\mathrm{s}(\mathrm{t})$ are not exactly known as they are assumed to be sinusoidal in nature based on a prior estimate, but there must be some algorithm that must be blind to the desired reference signal. Frequency selective channel diminish the property of constant amplitude, as there are certain modulation schemes that are having constant amplitude such as FM, PM, FSK and QPSK. If the incoming signal is of above nature of constant amplitude then adaptive beam forming must be one in accordance with that. This algorithm of constant modulus is called as blind equalization algorithm [14]. It uses a cost function based on incoming signal and thus error is calculated based on that cost function [14].

CMA cost function is given as

$$
\mathrm{J}(\mathrm{k})=\mathrm{E}\left[\left(|\mathrm{y}(\mathrm{k})|^{\mathrm{p}}-\mathrm{R}_{\mathrm{p}}\right)^{\mathrm{q}}\right]
$$

Where $\mathrm{p}$ and $\mathrm{q}$ are dispersion function and taking derivative of equation 14 gives the value of $R_{p}$.

$$
\mathrm{R}_{\mathrm{p}}=\mathrm{E}\left[(|\mathrm{s}(\mathrm{k})|)^{2 \mathrm{p}} /(|\mathrm{s}(\mathrm{k})|)^{\mathrm{p}}\right]
$$

$\mathrm{s}(\mathrm{k})$ is estimated incoming signal

$$
\begin{aligned}
& \mathrm{e}(\mathrm{k})=\text { error signal }=\mathrm{y}(\mathrm{k}) *|\mathrm{y}(\mathrm{k})|^{\mathrm{p}-2} *\left(\mathrm{R}_{\mathrm{p}}-|\mathrm{y}(\mathrm{k})|^{\mathrm{p}}\right) \\
& \mathrm{w}(\mathrm{k}+1)=\mathrm{w}(\mathrm{k})+\mu * \mathrm{e}^{*}(\mathrm{k}) * \mathrm{x}(\mathrm{k})
\end{aligned}
$$

\section{SIMULATION RESULTS}

Simulations are carried out on MATLAB ${ }^{\circledR}$ R2008b; if patch antenna considerations for array are taken in account the HFSS and IE3D simulation software can also be used.

First stage simulation includes DOA estimation using music algorithm. In Figure 3 MUSIC DOA results are shown desired arrival angles are taken as $+/-20^{\circ}$ degrees. These are compared with conventional DOA approach which surely has a low resolution. These results are shown for linear array or circular array geometry of $\mathrm{N}$ elements defined by the user; in this case $\mathrm{N}$ is taken 8 . Figure 4 presents the result for LMS adaptive beam-forming with 8 element uniform linear array with desired signal of interest at $+20^{\circ}$ degrees and interferer at $-60^{\circ}$ degrees, maximized peak of normalized array factor is obtained at desired angle. LMS also presents the dynamic change in array weights with the iterations in figure 5; this plot is for 8 element array which can be changed according to the user criteria.

Figure 6 and 7 depict the result for planner circular 8 elements and rectangular $8 * 8$ elements array respectively. These results are plotted with desired signal of interest at $+20^{\circ}$ degrees and interferer at $-60^{\circ}$ degrees. Deviation can be clearly seen from the figure 5 as the array factor changes for planner arrays. Figure 8 and 9 shows the results of RLS algorithm for 8 element array and with desired signal of interest at $+20^{\circ}$ degrees and interferer at $-60^{\circ}$ degrees. Faster convergence of array weights with respect to time samples can be visualized. RLS can be compared with LMS result of figure 4 which is much better for interferer rejection.

Figure 10 is the result of constant modulus algorithm with 8 elements array and desired angle at $+20^{\circ}$ degrees and two interfering signals at $-60^{\circ}$ degrees and $-80^{\circ}$ degrees; this algorithm works out with one direct path signal as 32 bit binary sequence and two multipath signal which are $20 \%$ and $10 \%$ of the direct path signal. $\mathrm{P}$ dispersion factor is taken as 1 . Figure 11 shows the combined constant modulus for these three signals. And also the arriving signal with respect to time.

Simulation results are carried out with certain assumptions such as incoming signal, wavelength and some other constraints. The effect is found to be very less as the aforesaid parameters is varied.

Equation 17 is weight update of CMA algorithm.

Equation ref.'s are from [12, 13, and 14] 


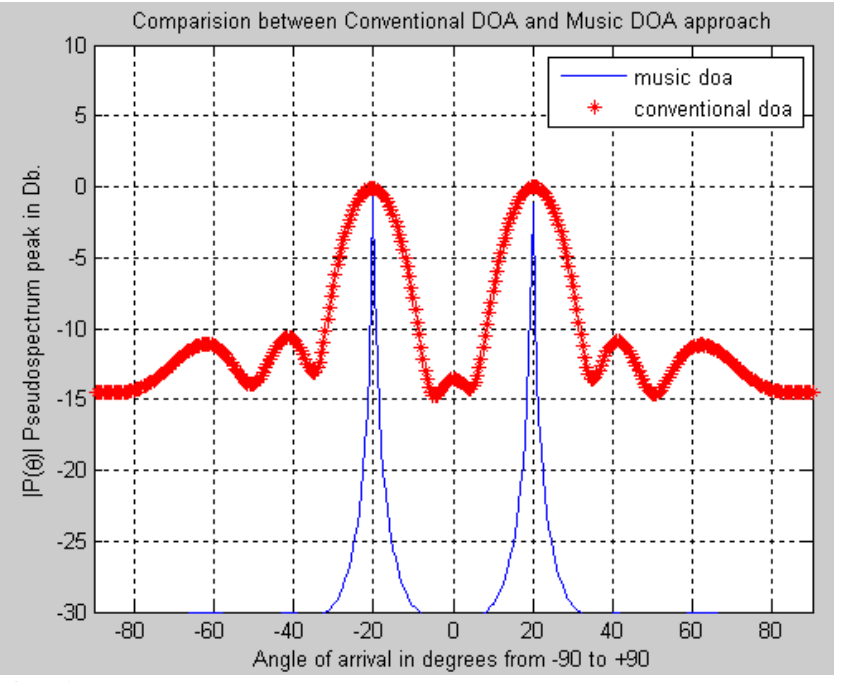

Fig- 3: MUSIC DOA estimation in solid line with 8 element array at +/- 20 degree arrival and its comparison with conventional DOA approach.

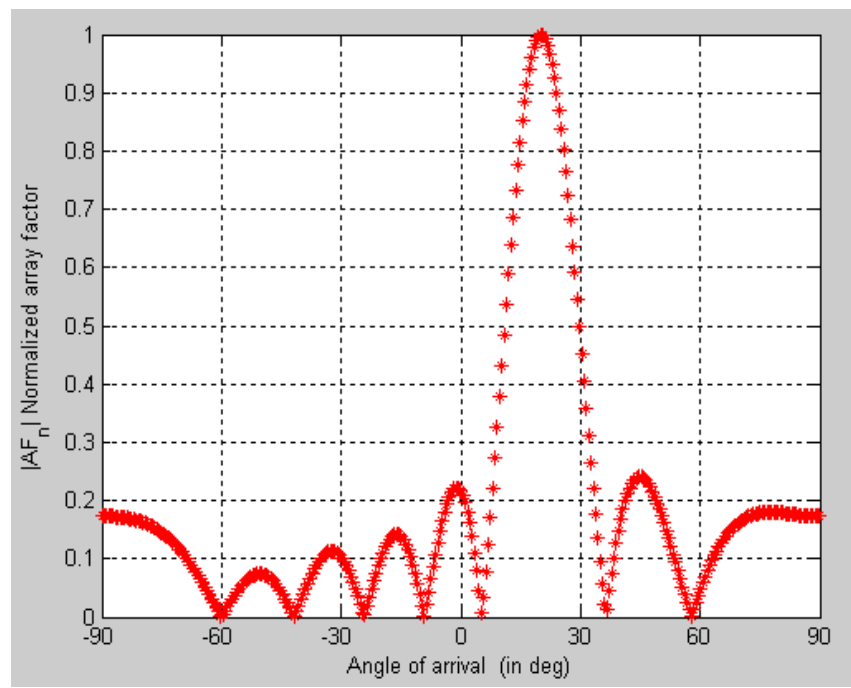

Fig- 4: LMS Beam-forming with 8 element array at +20 degree desired direction and -60 degree interferer direction

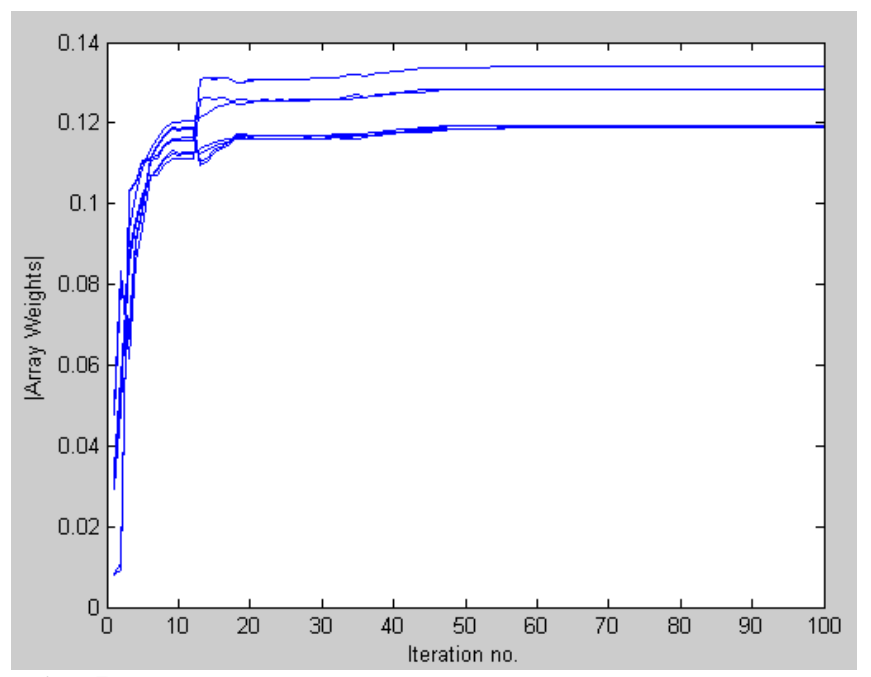

Fig- 5: LMS Beam-forming with 8 element array and array weights setting up with iterations.

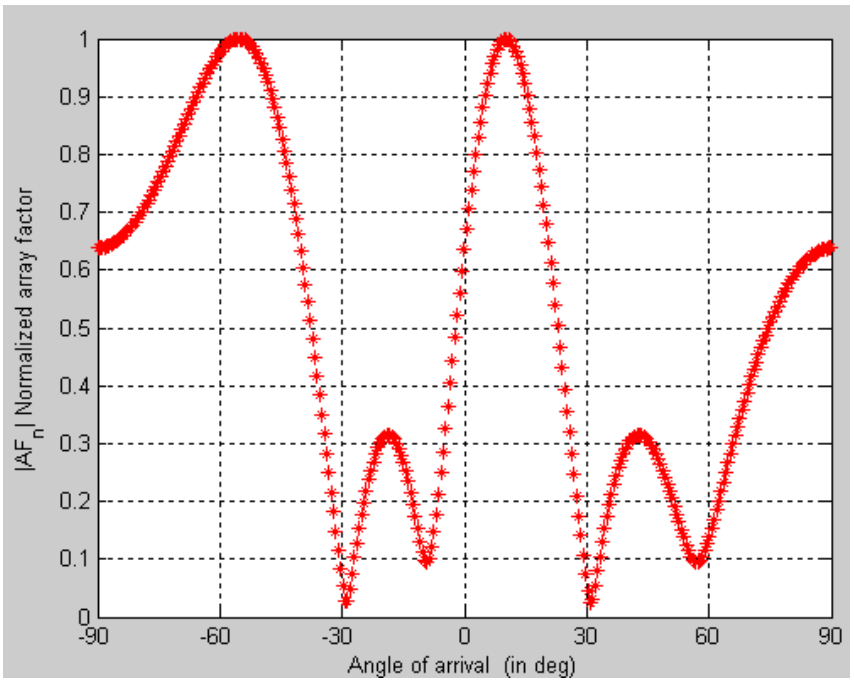

Fig- 6: LMS Beam-forming with 8 element uniform circular array at +20 degree desired direction and -60 degree interferer direction

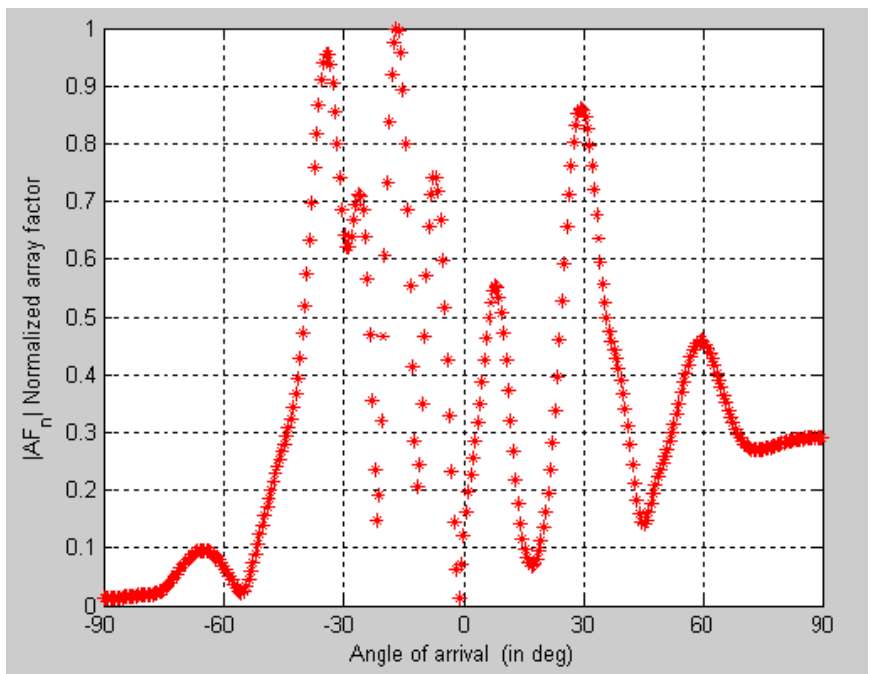

Fig- 7: LMS Beam-forming with $8 * 8$ element rectangular array at +20 degree desired direction and -60 degree interferer direction

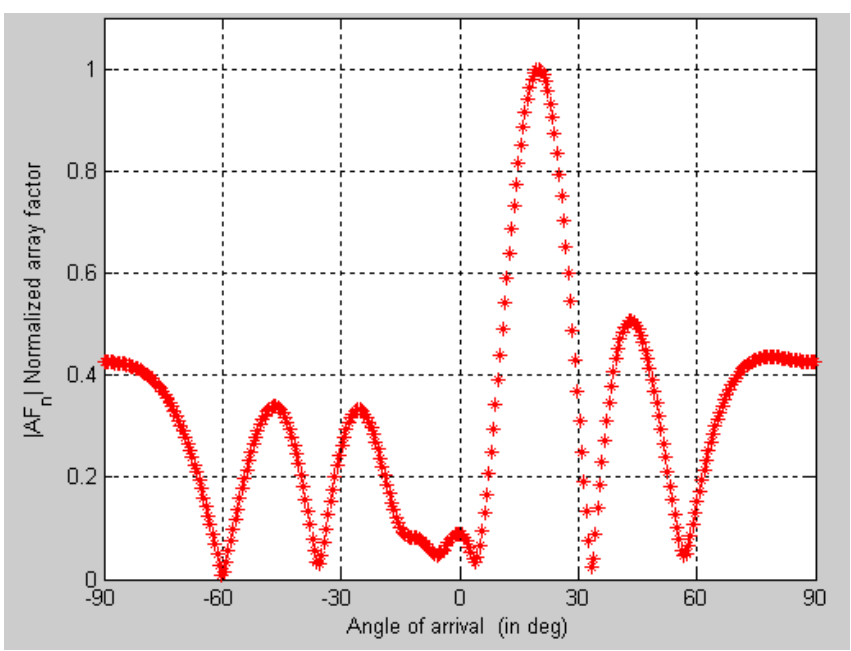

Fig- 8: RLS Beam-forming with 8 element array at +20 degree desired direction and -60 degree interferer direction 


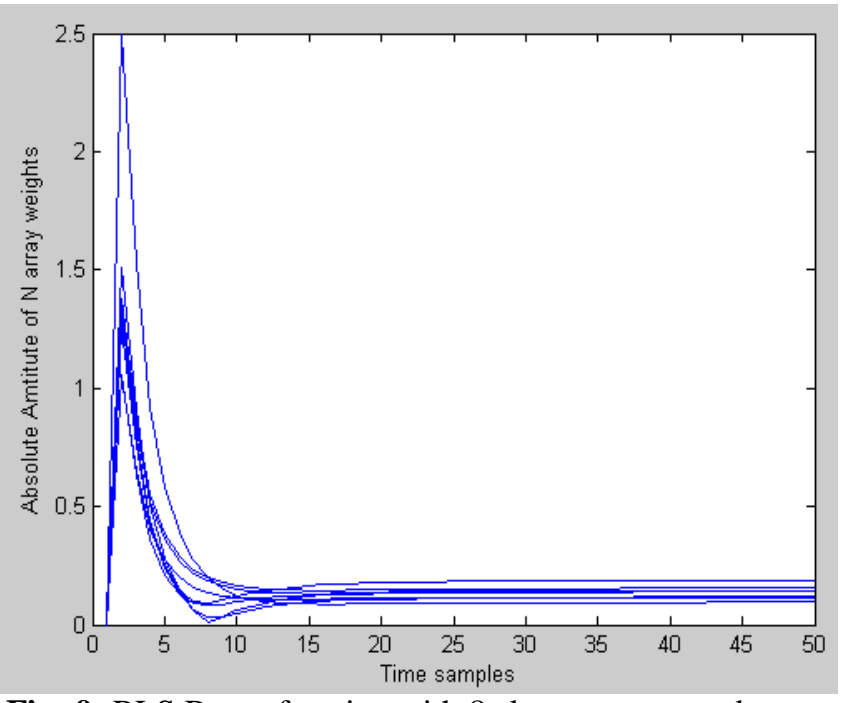

Fig- 9: RLS Beam-forming with 8 element array and array weights setting up with time-samples.

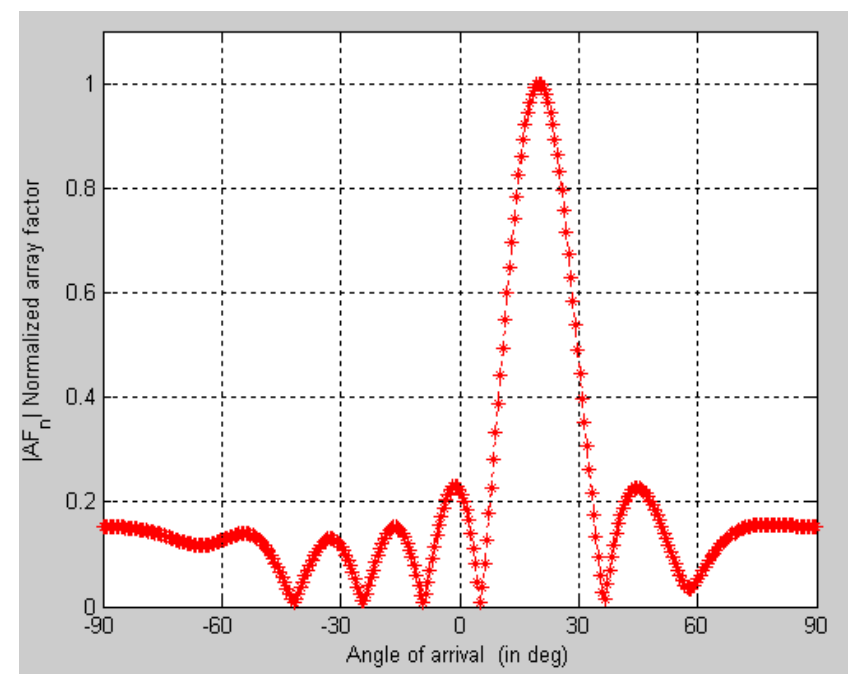

Fig- 10: CMA Beam-forming with 8 element array at +20 degree desired direction and -60 degree and -80 degree interferer direction

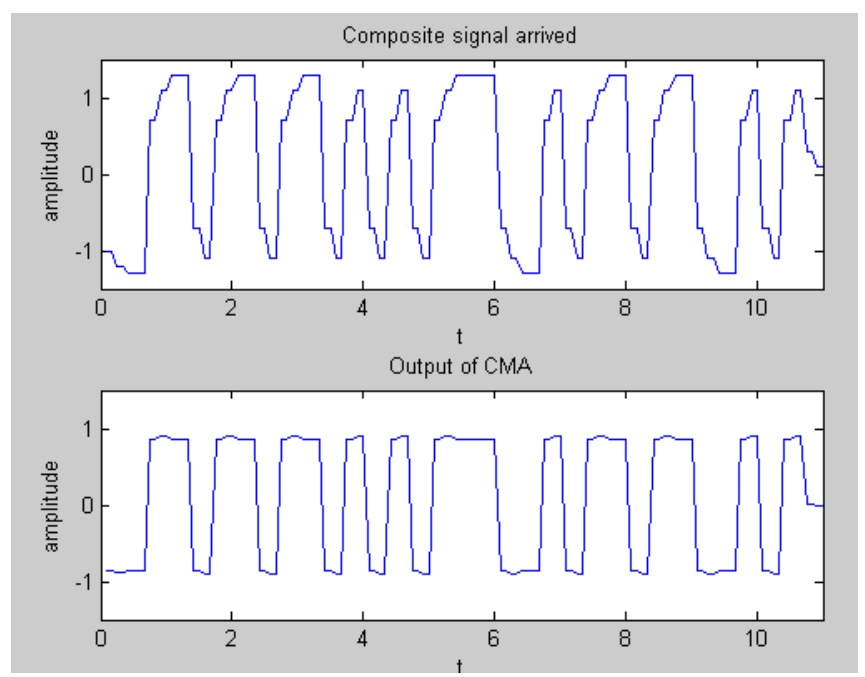

Fig- 11: Constant Modulus for combined 3 different signals $1^{\text {st }}$ signal with direct path $2^{\text {nd }}$ and $3^{\text {rd }}$ directional signals with $20 \%$ and $10 \%$ amplitude of the $1^{\text {st }}$.

\section{CONCLUSION}

In this paper DOA and adaptive beam-forming estimation is done on planner and linear geometry of arrays. Music algorithm has a sharp resolution for desired angle of interest as shown in figure, it is compared with conventional DOA approach, and Music is surely a better method with high resolution. LMS converges slowly in the dynamic environment with up to 60 iteration at a minimum. RLS solves this problem but RLS and CMA are not as better for rejecting interfering signals as LMS. But if convergence rate and speed is the major criteria then RLS and CMA are suitable. Convergence of CMA can be increased with increasing number of elements. Thus Smart antenna systems can be considered as the combined system of DOA and adaptive beam-forming for which algorithms are used in this paper.

\section{REFERENCES}

[1]. Dau- Chyrh Chang, Cheng Nan $\mathrm{Hu}$ - Smart Antennas for Advanced Communication Systems Vol. 100, No. 7, July 2012 Proceedings of the IEEE, 2012

[2]. RK Jain, Sumit Katiyar and NK Agrawal Smart Antenna for Cellular Mobile Communication Vol. 1(9), 530541, VSRD-IJEECE 2011

[3]. Younsun Kim, Hyoungju Ji, Hyojin Lee, Juho Lee, Boon Loong $\mathrm{Ng}$ and Jianzhong Zhang - Evolution beyond LTE-Advanced with Full Dimension MIMO - IEEE International Conference on communication 2013

[4]. Vira rahayu, Kei yokokawa - 2D DOA estimation of patch antenna array using USV MUSIC algorithm The Institute of Electronics Information and communication ICICE 2013

[5]. Afif Osseiran, Federico Boccardi, Volker Braun Scenarios for 5G Mobile and Wireless Communications: The Vision of the METIS Project IEEE Communication Magazine May 2014

[6]. D.B.Salunke, R.S.Kawaitkar, Analysis of LMS, NLMS and MUSIC Algorithms for Adaptive Array Antenna Systems, International Journal of Engineering and Advanced Technology (IJEAT) Vol. 2 Feb 2013

[7].Amara Prakasa Rao, N.V.S.N Sarma, Adaptive Beamforming algorithms for Smart Antenna Systems, WSEAS Transaction on Communications Vol.13 2014

[8].Amarnath Poluri, Ashish Kumar, Beam Steering in Smart Antennas by Using Low Complex Adaptive Algorithms, International Journal of Research in Engineering and Technology IJRET Vol. 2 Issue 10 Oct.2013

[9]. Sumit Katiyar, Prof. R. K. Jain, Prof. N. K. Agarwal, Proposed Cellular Network for Indian Conditions for Enhancement of Spectral Density and Reduction of Power Consumption \& RF Pollution, International Conference on Computer \& Communication Technology (ICCCT)-2011

[10]. Umar Mujahid, Jameel Ahmed, Mudassir Mukhtar, Abdul Rehman, Muhammad Abbas, Umair Shahid, Spectral Estimation for Smart Antenna System, 2013 IEEE

[11]. David Tse and Pramod Vishwanath - Fundamentals of Wireless Communication - Cambridge press 
[12]. Godara, Lal Chand - Handbook of Antennas in Wireless Communications - CRC press

[13]. Dimitris G. Manolakis and Stephen M. Kogon Statistical and Adaptive Signal Processing - Artech House

[14]. Frank B Gross Smart Antennas with Matlab - Mcgraw Hill publication.

\section{BIOGRAPHIES}

Amrita Soni: Amrita soni is asst. professor at Ujjain Engineering college Ujjain M.P. She is having M.Tech in Microwaves and $\mathrm{mm}$ waves. She is having teaching experience of 12 years. Her areas of interest include mobile communication, computer networks, antennas and wave propagation, analog and digital electronics. She is also the member of ISTE.

Mukesh Wadhwani: Mukesh wadhwani is pursuing M.E in electronics and communication from Ujjain Engineering College Ujjain M.P. He completed his B.E in ECE in 2010 and has 3 years of teaching experience. His areas of interest include Antennas, microwaves and wireless communication. 